John Carroll University

Carroll Collected

2019

\title{
Time savings and accuracy of a simulated flexible and conditional administration of the MMPI-2-RF in presurgical psychological evaluations of bariatric surgery candidates
}

Anthony M. Tarescavage

John Carroll University, atarescavage@jcu.edu

Yossef S. Ben-Porath

Kent State University

Ryan J. Marek

University of Houston, Clear Lake

Lana Boutacoff

Leslie J. Heinberg

Cleveland Clinic Lerner College of Medicine

Follow this and additional works at: https://collected.jcu.edu/fac_bib_2019

Part of the Psychology Commons

\section{Recommended Citation}

Tarescavage, Anthony M.; Ben-Porath, Yossef S.; Marek, Ryan J.; Boutacoff, Lana; and Heinberg, Leslie J., "Time savings and accuracy of a simulated flexible and conditional administration of the MMPI-2-RF in presurgical psychological evaluations of bariatric surgery candidates" (2019). 2019 Faculty Bibliography. 55.

https://collected.jcu.edu/fac_bib_2019/55

This Article is brought to you for free and open access by the Faculty Bibliographies Community Homepage at Carroll Collected. It has been accepted for inclusion in 2019 Faculty Bibliography by an authorized administrator of Carroll Collected. For more information, please contact connell@jcu.edu. 


\title{
Time savings and accuracy of a simulated flexible and conditional administration of the MMPI-2-RF in presurgical psychological evaluations of bariatric surgery candidates
}

\author{
Anthony M. Tarescavage, Ph.D., Yossef S. Ben-Porath, Ph.D., Ryan J. Marek, Ph.D., \\ Lana Boutacoff, Ph.D., Leslie J. Heinberg, Ph.D.
}

\begin{abstract}
Background: The Minnesota Multiphasic Personality Inventory-2-Restructured Form (MMPI-2RF) has empirically validated utility in presurgical psychological evaluations of bariatric surgery patients. However, clinicians may prefer shorter, symptom-focused measures.

Objectives: The purpose of the present study is to evaluate the feasibility and potential administration time savings of a proposed flexible and conditional (FCA) administration of the MMPI-2-RF in presurgical evaluations of bariatric surgery candidates.

Setting: Bariatric surgery candidates evaluated at a large hospital as well as a private practice in the Midwest.

Methods: MMPI-2-RF scores were available for a total of 4099 adult bariatric surgery candidates from 2 separate samples. The hospital sample included 911 males and 2430 females. The average BMI was $49.3 \mathrm{~kg} / \mathrm{m}^{2}(S D=11.0)$. The private practice sample included 105 males, 640 females, and 13 individuals who did not report gender. The average BMI was $48.8 \mathrm{~kg} / \mathrm{m}^{2}(S D=8.4)$. The authors used a simulation design in which existing MMPI-2-RF responses were used to simulate an FCA administration.

Results: The findings indicated that an FCA of the MMPI-2-RF closely approximates the amount of information typically gained from a full administration of the test in the 2 samples of bariatric surgery candidates. Items savings and estimated time savings ranged from $44 \%$ to $88 \%$ in both samples, depending on the number of conditionally administered scales.

Conclusions: The present study supports the feasibility of an FCA of the MMPI-2-RF, potentially shortening administration time and reducing patient burden. However, the findings are limited because the accuracy and time savings are based on a simulation, not actual FCA administrations. (Surg Obes Relat Dis 2019;15:732-738.) (C) 2019 American Society for Bariatric Surgery. Published by Elsevier Inc. All rights reserved.
\end{abstract}

Broadband psychological tests like the Minnesota Multiphasic Personality Inventory-2-Restructured Form (MMPI-2-RF) [1] are recommended for use in presurgical psychological assessments of bariatric surgery patients 
[2] because of research literature supporting the utility of the test in this setting [3-6]. For example, Marek et al. [5] found that scores from the test predicted appointment adherence and weight loss outcomes 1 year postsurgery. Nevertheless, psychologists conducting these evaluations tend to administer shorter, more symptom-focused measures rather than broadband instruments with longer administration times [7]. Although the reasons for this trend among psychologists conducting presurgical evaluations have not been directly studied, research with child psychologists indicates that concerns about the practicality of assessment instruments (e.g., particularly patient burden as well as administration, scoring, and interpretation time) are negatively associated with psychological test usage [8]. However, recent efforts to decrease MMPI-2-RF administration time could increase use of broadband personality testing in presurgical evaluations.

Tarescavage and Ben-Porath [9] have proposed and evaluated the feasibility of a flexible and conditional administration (FCA) of the MMPI-2-RF intended to decrease administration time. In an FCA framework, high scores on scales measuring broad areas of psychopathology are used to trigger administration of lower-level scales measuring more specific symptoms of psychological problems in that domain. For example, a high score on a measure of disordered thinking can trigger administration of measures of hallucinations and delusions. In the context of the MMPI-2-RF, scores above a predetermined cutoff on the higher-order scale Thought Dysfunction (THD) would trigger administration of the lower-level scales in the thought dysfunction domain (i.e., Ideas of Persecution [RC6], Aberrant Experiences [RC8], and Psychoticism [PSYC-r]). The method is flexible in that clinicians can choose which broad domains are conditionally assessed (e.g., thought, mood, behavior, and somatic/cognitive problems). It also incorporates the over- and underreporting validity scales of the MMPI-2-RF.

Tarescavage and Ben-Porath [9] derived rules for and evaluated the FCA approach using a real-data simulation in the MMPI-2-RF Normative Sample and the Community Mental Health Center comparison group. They found that the FCA method resulted in minimal information loss in both settings. Potential time savings were much more pronounced in the normative sample, in which estimated administration times were 4 to 20 minutes depending on the number of domains assessed. The authors concluded that an FCA of the MMPI-2-RF held promise in settings with time constraints and relatively low base rates of psychopathology (compared to mental health treatment settings), such as bariatric surgery patients. For these reasons, the purpose of this study is to describe, derive rules for, and evaluate an FCA of the MMPI-2-RF by using existing data to simulate FCA administrations with over 4000 bariatric surgery candidates.

\section{Method}

\section{Participants}

The authors used samples of 3341 and 758 patients who received presurgical psychological evaluations at a large hospital in Cleveland, Ohio, and a private practice in St. Paul, Minnesota, respectively. De-identified and aggregated use of this existing archival clinical information was approved by the Kent State University Institutional Review Board before manuscript submission.

The hospital sample included 911 males and 2430 females. The majority of patients identified as white (68.3\%), with $23.3 \%$ identifying as black and $3.2 \%$ identifying as Hispanic/Latino. The most common marital statuses were married $(53.1 \%)$, never married $(24.5 \%)$, and divorced $(16.3 \%)$. The average years of education was 14.0 (standard deviation $[\mathrm{SD}]=2.9$ ). Intake BMI was available for 1411 patients. The average BMI was $49.3 \mathrm{~kg} / \mathrm{m}^{2}$ $(\mathrm{SD}=11.0)$. Age was available for 1452 participants, with the average being 46.2 years $(\mathrm{SD}=11.6)$. A total of 132 (4\%) patients produced invalid MMPI-2-RF protocols according to standard interpretive guidelines outlined by BenPorath and Tellegen [1].

The private practice sample included 105 males, 640 females, and 13 individuals who did not report gender. The majority of patients identified as white $(86.9 \%)$, with 9.2\% identifying as black. The most common marital statuses were married (49.7\%), never married $(29.7 \%)$, and divorced (20.6\%). In terms of educational levels, the majority of the sample reported some college $(53.1 \%)$, with $20.1 \%$ reporting being a high school graduate, $16.5 \%$ reporting having a bachelor's degree, and $8.4 \%$ reporting at least some postgraduate education. Intake BMI was available for 209 patients. The average BMI was $48.8 \mathrm{~kg} / \mathrm{m}^{2}$ $(\mathrm{SD}=8.4)$. Age was available for 325 participants, with the average being 38.7 years $(\mathrm{SD}=10.4)$. A total of $13(2 \%)$ patients produced invalid MMPI-2-RF protocols according to standard interpretive guidelines.

Although specific information on the surgical approval rates from psychological evaluations was not available for the study samples, a study using a subsample from this study's hospital sample found that only $2.6 \%$ of candidates were deemed unlikely to meet the psychological requirements for surgery [10].

\section{Measure}

The MMPI-2-RF is a 338 item true-false measure of response style (e.g., overreporting and underreporting) and 5 broad substantive domains of psychopathology (emotional, thought, behavior, somatic/cognitive, and interpersonal). Four of the 5 domains of substantive scales are organized hierarchically, whereas the interpersonal functioning domain does not have a hierarchical structure. The measures at the broadest level of the hierarchy for the 4 other 
domains are the higher-order scales Emotional Internalizing Dysfunction (EID), THD, Behavioral/Externalizing Dysfunction (BXD), and the Restructured Clinical Scale Somatic Complaints (RC1). Interpretive cutoffs for the measures of overreporting response style (overreporting validity scales) generally range from $80 \mathrm{~T}$ to $120 \mathrm{~T}$. The cutoff for a clinically significant elevation is $65 \mathrm{~T}$ on the substantive scales of the test, which measure the just mentioned 5 domains of psychopathology. Psychometric information is provided in the test's technical manual [11] and reviewed by Ben-Porath [12].

\section{Procedure}

\section{FCA Protocol}

A detailed description of the FCA framework is provided by Tarescavage and Ben-Porath [9]. Briefly, in an FCA with the MMPI-2-RF, the clinician selects 1 to 4 substantive domains to administer (emotion, thought, behavior, and somatic/cognitive). The EID, THD, BXD, and $\mathrm{RC} 1$ scales are administered, depending on the selected domains. If a test-taker scores above a predetermined cutoff on a broad domain, this triggers administration of lowerlevel scales from that domain. The overreporting validity scales can also be administered in an FCA of the MMPI-2RF. The MMPI-2-RF underreporting scales (L-r and K-r) are always administered.

Cutoffs for triggering administration of lower-level scales (i.e., administration rules) are optimized by setting. For bariatric surgery candidates, the authors identified administration cutoff rules in a subsample of 1941 bariatric surgery patients from a large, Midwestern hospital using a real-data simulation design with MMPI-2-RF items. In this approach, existing data are used to simulate FCA administrations. Administration rules are derived by observing and optimizing clinical elevation catch rates among lower-level substantive scales and the overreporting validity indicators. The catch rate is the rate at which a clinically elevated scale would have been conditionally administered (and thus found to be elevated) according to the FCA administration rules. For example, if 100 test-takers in a sample have clinically elevated scores on RC6, and $90 \%$ of these cases are administered RC6 by the administration rule derived for THD, then the RC6 catch rate would be $90 \%$.

The authors reduced the triggering scale administration rules from $65 \mathrm{~T}$ to $60 \mathrm{~T}$ and to 55T until the lower-level scale catch rates were at least $80 \%$ in the derivation sample. If a cutoff of 55T for a triggering scale did not result in an adequate elevation catch rate, the authors decreased the administration condition by smaller increments than $5 \mathrm{~T}$ because these scores are in the normative range where small decreases in cutoff rules could substantially increase the number of individuals administered a lower-order scale. Supplementary Table 1 has the resulting cutoff rules and sample descriptive statistics.

\section{Statistical analyses}

The authors conducted an evaluation of the FCA rules in the private practice sample $(n=758)$ and the subsample of 1311 bariatric surgery patients from the large hospital who were not used to develop administration rules (i.e., these were cross-validation samples). For each scale, the authors calculated clinical elevation catch rates (described earlier in this section) as well as overall accuracy rates, which indicate the overall percentage of FCA administrations that would yield the same interpretive information as a standard administration.

The meaning of catch rate can be illustrated by using the hypothetical RC6 example described earlier in this section. Assume a sample size of 1000 . As noted earlier, 100 people had an elevated RC6 score. This means that 900 people produced unelevated scores on RC6. Of the 100 people who had an elevated RC6 score, the items from that scale were conditionally administered to 90 test-takers (i.e., catch rate $=90 \%$ ). The overall accuracy rate for RC6 equals the number of unelevated RC6 scores + number of caught RC6 elevated scores / overall sample size: $(900+90) / 1000=99 \%$. In other words, for 99\% of cases, an FCA of the MMPI-2-RF thought dysfunction domain would yield the same interpretive information as a standard administration in terms of identification of high-score elevations.

The authors calculated catch rates and overall accuracy rates for the substantive scales at elevations of 65T, 70T, and $80 \mathrm{~T}$ and for the validity scales (which require higher cutoffs) at $80 \mathrm{~T}, 100 \mathrm{~T}$, and $120 \mathrm{~T}$. The authors also estimated the median administration time savings of the FCA in the evaluation samples by examining the difference between the median number of FCA-administered items relative to a full MMPI-2-RF administration, which has a 25 to 35 minute computer administration time [1].

\section{Results}

\section{Elevation catch rates and overall accuracy rates}

The authors first examined elevation catch rates and overall accuracy rates among the overreporting validity scales (see Supplementary Table 1 for administration rules and Table 1 for results). In the hospital evaluation sample, the median catch rate for elevations at $80 \mathrm{~T}$ was $91.7 \%$. In the case of Fp-r (catch rate $=91.7 \%$ ), this means that the scale was administered in $91.7 \%$ of cases where an elevation of $80 \mathrm{~T}$ would have occurred. The median $80 \mathrm{~T}$ overall accuracy rate in the hospital evaluation sample was $99.7 \%$. For example, FBS-r had an overall accuracy rate of $99.7 \%$. This indicates that the FCA approach yielded the same FBS-r interpretive information as a full administration in $99.7 \%$ of cases (in terms of identifying elevations at $80 \mathrm{~T}$ or higher). At cutoffs of 100T and 120T, the median catch rates were both $100 \%$ in the hospital sample and private 
Table 1

Validity and substantive scales elevation catch rates in evaluation samples.

\begin{tabular}{|c|c|c|c|c|c|c|c|c|c|c|c|c|}
\hline \multirow{3}{*}{$\begin{array}{l}\text { Validity } \\
\text { scales }\end{array}$} & \multicolumn{6}{|c|}{ Hospital validation sample $(n=1272)$} & \multicolumn{6}{|c|}{ Private practice sample $(n=745)$} \\
\hline & \multicolumn{2}{|l|}{$80 \mathrm{~T}(\%)$} & \multicolumn{2}{|l|}{$100 \mathrm{~T}(\%)$} & \multicolumn{2}{|l|}{$120 \mathrm{~T}(\%)$} & \multicolumn{2}{|l|}{$80 \mathrm{~T}(\%)$} & \multicolumn{2}{|l|}{$100 \mathrm{~T}(\%)$} & \multicolumn{2}{|l|}{$120 \mathrm{~T}(\%)$} \\
\hline & Catch rate & OVRACC & Catch rate & OVRACC & Catch rate & OVRACC & Catch rate & OVRACC & Catch rate & OVRACC & Catch rate & OVRACC \\
\hline F-r & 97.8 & 99.9 & 100 & 100 & 100 & 100 & 95.6 & 97.9 & 99.6 & 99.9 & 100 & 100 \\
\hline $\mathrm{F}_{\mathrm{P}-\mathrm{r}}$ & 91.7 & 99.9 & 100 & 100 & 100 & 100 & 99.6 & 99.9 & 100 & 100 & 100 & 100 \\
\hline $\mathrm{F}_{\mathrm{S}}$ & 89.5 & 99.4 & 100 & 100 & 100 & 100 & 87.7 & 97.6 & 98.6 & 99.7 & 100 & 100 \\
\hline FBS-r & 94 & 99.7 & 100 & 100 & 100 & 100 & 80.1 & 96.1 & 100 & 100 & 100 & 100 \\
\hline RBS & 90.2 & 99.5 & 100 & 100 & 100 & 100 & 89.4 & 97.9 & 100 & 100 & 100 & 100 \\
\hline L-r & 100 & 100 & 100 & 100 & 100 & 100 & 100 & 100 & 100 & 100 & 100 & 100 \\
\hline K-r & 100 & 100 & 100 & 100 & 100 & 100 & 100 & 100 & 100 & 100 & 100 & 100 \\
\hline \multirow{3}{*}{$\begin{array}{l}\text { Substantive } \\
\text { scales }\end{array}$} & \multicolumn{6}{|c|}{ Hospital validation sample $(n=1268)$} & \multicolumn{6}{|c|}{ Private practice sample $(n=745)$} \\
\hline & \multicolumn{2}{|l|}{$65 \mathrm{~T}(\%)$} & \multicolumn{2}{|l|}{$70 \mathrm{~T}(\%)$} & \multicolumn{2}{|l|}{$80 \mathrm{~T}(\%)$} & \multicolumn{2}{|l|}{$65 \mathrm{~T}(\%)$} & \multicolumn{2}{|l|}{$70 \mathrm{~T}(\%)$} & \multicolumn{2}{|l|}{$80 \mathrm{~T}(\%)$} \\
\hline & Catch rate & OVRACC & Catch rate & OVRACC & Catch rate & OVRACC & Catch rate & OVRACC & Catch rate & OVRACC & Catch rate & OVRACC \\
\hline EID & 100 & 100 & 100 & 100 & 100 & 100 & 100 & 100 & 100 & 100 & 100 & 100 \\
\hline THD & 100 & 100 & 100 & 100 & 100 & 100 & 100 & 100 & 100 & 100 & 100 & 100 \\
\hline BXD & 100 & 100 & 100 & 100 & 100 & 100 & 100 & 100 & 100 & 100 & 100 & 100 \\
\hline $\mathrm{RCd}$ & 81.3 & 97.7 & 96.3 & 99.8 & 100 & 100 & 80.0 & 97.6 & 93.5 & 99.6 & 100 & 100 \\
\hline $\mathrm{RC} 1$ & 100 & 100 & 100 & 100 & 100 & 100 & 100 & 100 & 100 & 100 & 100 & 100 \\
\hline $\mathrm{RC} 2$ & 84.6 & 97.6 & 93.1 & 99.5 & 97.4 & 99.9 & 86.1 & 98.0 & 92.0 & 99.5 & 100 & 100 \\
\hline $\mathrm{RC} 4$ & 92.2 & 99.7 & 94.7 & 99.9 & 100 & 100 & 98.1 & 99.9 & 96.0 & 99.9 & 100 & 100 \\
\hline RC6 & 91.8 & 99.1 & 93.5 & 99.6 & 100 & 100 & 82.8 & 98.5 & 89.7 & 99.6 & 100 & 100 \\
\hline $\mathrm{RC7}$ & 76.8 & 98.5 & 86.7 & 99.5 & 100 & 100 & 75.0 & 98.0 & 86.2 & 99.5 & 100 & 100 \\
\hline RC8 & 98.3 & 99.9 & 100 & 100 & 100 & 100 & 100 & 100 & 100 & 100 & 100 & 100 \\
\hline RC9 & 86.2 & 99.7 & 100 & 99.9 & 100 & 100 & 87.5 & 99.7 & 100 & 100 & 100 & 100 \\
\hline MLS & 80.4 & 91.0 & 86.7 & 95.8 & 94.8 & 99.2 & 76.4 & 89.7 & 83.6 & 96.1 & 93.3 & 99.3 \\
\hline GIC & 100 & 100 & 100 & 100 & 100 & 100 & 100 & 100 & 100 & 100 & 100 & 100 \\
\hline HPC & 100 & 100 & 100 & 100 & 100 & 100 & 100 & 100 & 100 & 100 & 100 & 100 \\
\hline NUC & 100 & 100 & 100 & 100 & 100 & 100 & 100 & 100 & 100 & 100 & 100 & 100 \\
\hline $\mathrm{COG}$ & 90.1 & 98.9 & 91.3 & 99.4 & 100 & 99.9 & 89.4 & 99.3 & 95.2 & 99.9 & 100 & 99.9 \\
\hline SUI & 100 & 100 & 100 & 100 & 100 & 100 & 100 & 100 & 100 & 100 & 100 & 100 \\
\hline HLP & 90.8 & 99.5 & 100 & 100 & 100 & 100 & 88.2 & 99.7 & 100 & 100 & 100 & 100 \\
\hline SFD & 100 & 100 & 100 & 100 & $\mathrm{n} / \mathrm{a}$ & $\mathrm{n} / \mathrm{a}$ & 100 & 100 & 100 & 100 & $\mathrm{n} / \mathrm{a}$ & $\mathrm{n} / \mathrm{a}$ \\
\hline NFC & 76.6 & 98.3 & 84.0 & 99.4 & 86.7 & 99.8 & 77.6 & 98.5 & 88.5 & 99.6 & 84.6 & 99.7 \\
\hline STW & 82.6 & 97.5 & 96.3 & 99.8 & 100 & 100 & 94.4 & 99.5 & 100 & 100 & 100 & 100 \\
\hline AXY & 90.0 & 99.1 & 90.0 & 99.1 & 95.4 & 99.8 & 88.2 & 98.9 & 88.2 & 98.9 & 100 & 100 \\
\hline ANP & 73.8 & 97.5 & 76.8 & 99.0 & 71.4 & 99.7 & 78.8 & 98.1 & 93.9 & 99.7 & 91.7 & 99.9 \\
\hline BRF & 83.0 & 99.3 & 83.0 & 99.3 & 71.4 & 99.8 & 79.2 & 99.3 & 79.2 & 99.3 & 100 & 100 \\
\hline MSF & 65.1 & 96.5 & 63.3 & 98.6 & $\mathrm{n} / \mathrm{a}$ & $\mathrm{n} / \mathrm{a}$ & 65.8 & 96.4 & 57.7 & 98.5 & $\mathrm{n} / \mathrm{a}$ & $\mathrm{n} / \mathrm{a}$ \\
\hline JCP & 100 & 100 & 100 & 100 & 100 & 100 & 100 & 100 & 100 & 100 & 100 & 100 \\
\hline SUB & 100 & 100 & 100 & 100 & 100 & 100 & 100 & 100 & 100 & 100 & 100 & 100 \\
\hline AGG & 87.5 & 99.7 & 85.7 & 99.8 & 100 & 100 & 86.7 & 99.7 & 100 & 100 & 100 & 100 \\
\hline $\mathrm{ACT}$ & 65.5 & 98.4 & 80.0 & 99.7 & 100 & 100 & 77.4 & 98.4 & 88.0 & 99.6 & 50.0 & 99.9 \\
\hline AGGR-r & 90.0 & 99.1 & 96.0 & 99.9 & 100 & 100 & 88.9 & 99.3 & 100 & 100 & 100 & 100 \\
\hline PSYC-r & 100 & 100 & 100 & 100 & 100 & 100 & 100 & 100 & 100 & 100 & 100 & 100 \\
\hline DISC-r & 80.0 & 99.6 & 100 & 100 & 100 & 100 & 90.6 & 99.6 & 100 & 100 & 100 & 100 \\
\hline NEGE-r & 79.6 & 98.2 & 89.6 & 99.6 & 100 & 100 & 83.3 & 98.5 & 96.3 & 99.9 & 100 & 100 \\
\hline INTR-r & 83.2 & 97.5 & 89.8 & 98.9 & 100 & 100 & 88.9 & 98.4 & 90.0 & 98.9 & 100 & 100 \\
\hline
\end{tabular}

Catch rate $=$ percent of elevated cases that were conditionally administered the scale; OVR ACC $=$ overall accuracy rate: percent of all cases that were [a] conditionally administered the scale when an elevation occurred [Catch Rate] or [b] did not produce an elevation on the scale, regardless of whether the

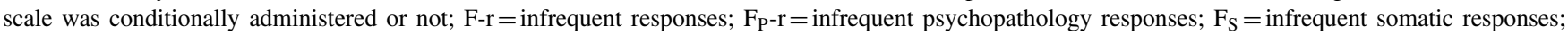
FBS-r = symptom validity; $\mathrm{L}-\mathrm{r}=$ uncommon virtues; $\mathrm{K}-\mathrm{r}=$ adjustment validity; $\mathrm{n} / \mathrm{a}=$ score not possible; $\mathrm{MH}=$ mental health; EID = emotional/internalizing dysfunction; $\mathrm{THD}=$ thought dysfunction; $\mathrm{BXD}=$ behavioral/externalizing dysfunction; $\mathrm{RCd}=$ demoralization; $\mathrm{RC} 1=$ somatic complaints; $\mathrm{RC} 2=$ low positive emotions; $\mathrm{RC} 4=$ antisocial behavior; $\mathrm{RC} 6=$ ideas of persecution; $\mathrm{RC} 7=$ dysfunctional negative emotions; $\mathrm{RC} 8=\mathrm{aberrant}$ experiences; RC9=hypomanic activation; MLS=malaise; GIC gastrointestinal complaints; HPC $=$ head pain complaints; NUC $=$ neurologic complaints; $\mathrm{COG}=$ cognitive complaints; $\mathrm{SUI}=$ suicidal/death ideation; HLP = helplessness/hopelessness; $\mathrm{SFD}=$ self-doubt; NFC $=$ inefficacy; $\mathrm{STW}=$ stress/worry; $\mathrm{AXY}=$ anxiety; $\mathrm{ANP}=$ anger proneness; $\mathrm{BRF}=$ behavior-restricting fears; $\mathrm{MSF}=$ multiple specific fears; JCP $=$ juvenile conduct problems; $\mathrm{SUB}=$ substance abuse; $\mathrm{AGG}=$ aggression; $\mathrm{ACT}=$ activation; AGGR- $\mathrm{r}=$ aggressiveness-revised; PSYC $-\mathrm{r}=$ psychoticism-revised; DISC- $\mathrm{r}=$ disconstraint revised; NEGE-r = negative emotionality/neuroticism-revised; INTR-r =introversion/low positive emotionality-revised.

Administration rules developed in hospital development sample $(\mathrm{n}=1941)$ For validity scale analyses, individuals excluded if CNS $>18$, VRIN-r $\geq$ $80 \mathrm{~T}$, or TRIN-r $\geq 80 \mathrm{~T}$. 
Table 2

Item savings in hospital evaluation sample $(n=1311)$ and private practice sample $(n=758)$.

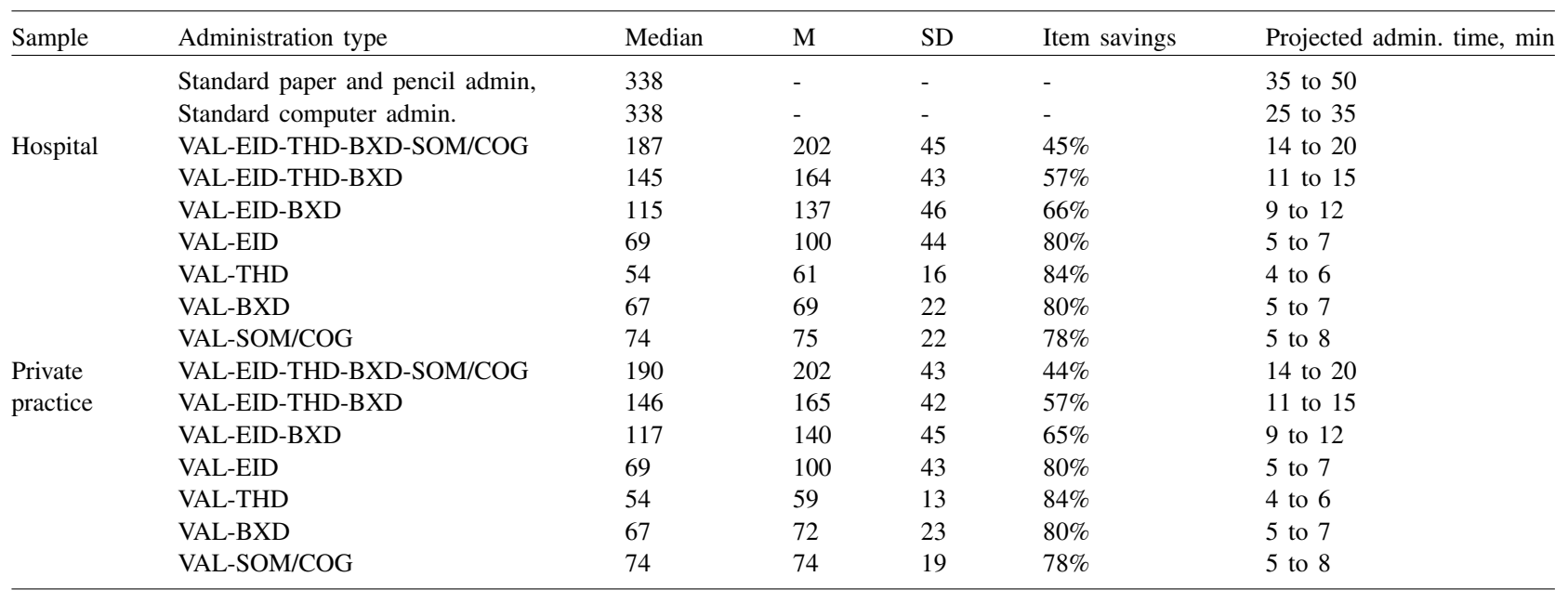

Admin. = administration; $\quad$ VAL = validity scales; $\quad$ EID = emotional dysfunction domain; THD = thought dysfunction $\quad$ domain; $\mathrm{BXD}=$ behavioral/externalizing dysfunction domain; SOM/COG = somatic/cognitive complaints domain).

Projected administration times based on estimate of a 25 to 35 min computer administration for the full 338 items, as described in the MMPI-2-RF administration, scoring, and interpretation manual [1].

practice sample. In the private practice sample, the median catch rate was $89.4 \%$ and median overall accuracy rate was $97.9 \%$ for identifying validity scale elevations of $80 \mathrm{~T}$.

In Table 1 the authors present catch rates and overall accuracy miss rates for substantive scales with a conditional administration. In the hospital evaluation sample, the median catch rate for elevations at $65 \mathrm{~T}$ was $83.2 \%$ and the median overall accuracy rate was $98.9 \%$. At a cutoff of $70 \mathrm{~T}$, the median catch rate was $91.3 \%$ and the overall accuracy rate was $99.6 \%$. At a cutoff of $80 \mathrm{~T}$, both the median catch rate and overall accuracy rate were $100 \%$ in the hospital sample and private practice sample. In the private practice sample, the median catch rate for elevations at $65 \mathrm{~T}$ was $86.7 \%$ and the median overall accuracy rate was $98.9 \%$. At a cutoff of $70 \mathrm{~T}$, the median catch rate was $93.9 \%$ and the overall accuracy rate was $99.7 \%$.

Overall, these findings indicate that an FCA of the MMPI-2-RF would closely approximate the information typically gained from a full administration of the scales included in the FCA procedure in 2 samples of bariatric surgery candidates. Next, analyses are presented comparing estimated administration time for an FCA and standard administration of the MMPI-2-RF in these 2 samples.

\section{Item and time savings}

In Table 2, the authors present the median item savings and estimated time savings of the standard computer administration of the MMPI-2-RF versus 7 possible FCAs in the study samples. To orient the reader to the statistics, the authors describe next the item and time savings for an FCA of the validity scales EID, THD, BXD, and Somatic/Cognitive Complaints Domain domains of the test in the hospital evaluation sample (i.e., the third row of Table 2). The median of 187 indicates that in a typical administration a total of 187 items were administered. This represents an absolute item decrease of $45 \%$ compared with the standard 338 item administration. By extension, a $45 \%$ decrease of the typical 338 item administration time (25 to $35 \mathrm{~min}$ [1]) yields a projected computer administration time of 14 to 20 minutes. Of note, because the distributions of items administered were positively skewed, the median is a better estimate of typical time savings (i.e., of the center of the distribution).

Overall, estimated savings were high in both samples, particularly when fewer substantive domains were administered. For example, if only the validity scales and the EID domain were selected for administration, the median number of items administered was 69 in both samples. This represented an $80 \%$ decrease in the number of administered items relative to a standard administration. Overall, the projected administration time in this case would be 5 to 7 minutes.

\section{Discussion}

The purpose of the present study was to explore flexible conditional administration of the MMPI-2-RF with 2 large samples of bariatric surgery candidates. The authors found that the rules developed in a subsample of candidates evaluated at a large hospital cross-validated well to a different subsample from the same large hospital and a sample from a private practice. Estimated time savings were high, as an FCA of the MMPI-2-RF had $44 \%$ to $88 \%$ decreases in test administration time depending on the number of conditionally administered scales. Whereas a paper-and-pencil administration of the MMPI-2-RF takes 35 to 50 minutes, 
the projected FCA administration times ranged from 4 to 20 minutes.

One possible application of an FCA of the MMPI-2$\mathrm{RF}$ in bariatric surgery candidates is to screen all bariatric surgery candidates, who present with a wide-array of psychological problems $[13,14]$. For example, Mitchell et al. [15] examined a sample of 199 bariatric surgery candidates and found that $33.7 \%$ had a psychiatric disorder and $11.6 \%$ had an affective disorder. Patients met diagnostic criteria for a wide variety of psychological problems, including major depressive disorder, dysthymia, social phobia, specific phobia, posttraumatic stress disorder, substance use disorders, and eating disorders. In an earlier study, Kalarchian et al. [14] examined a similarly sized sample and found that patients also met criteria for bipolar disorder, panic disorder, agoraphobia, obsessive-compulsive disorder, and personality disorders from all clusters. A full FCA of the MMPI-2-RF could provide information about psychological constructs related to all of these disorders in an estimated administration time of 14 to 20 minutes (e.g., by way of scales such as low positive emotions, dysfunctional negative emotions, hypomanic activation). These time savings will not only decrease patient burden but may also increase clinician productivity. For example, an FCA approach may enable administering testing on the same day as the clinical interview, thus facilitating the overall intake process. However, some clinicians already using the test may not find much benefit from an approximate 15minute time-savings, particularly given that some elevations may not be caught. Nevertheless, for those clinicians not using the test because of time constraints, $100 \%$ of elevations on the MMPI-2-RF are necessarily missed at this juncture.

Another possible application of an FCA with bariatric surgery candidates is for more focused diagnostic assessment. As noted, the most common diagnoses among bariatric surgery candidates are mood and anxiety disorders. One commonly used option to assess these disorders is to administer 2 short, symptom-focused measures of depression and anxiety, which might take 5 to 10 minutes to administer. However, the results of this study suggest that an FCA of the MMPI-2-RF emotional dysfunction domain items (along with the MMPI-2-RF underreporting indicators) would take approximately 5 to 7 minutes. In contrast to the typical approach, a total of 19 areas of emotional dysfunction and response style assessment (which is not measured by symptom-focused measures) would be covered by this FCA administration. Of note, assessing response styles among bariatric surgery candidates is especially important because they commonly underreport psychological problems [16]. Of note, a limitation of the FCA protocol is that meaningful but subthreshold (i.e., $<65 \mathrm{~T}$ ) scores may not be available because of underreporting. Nevertheless, broad upper-level scales such as EID, BXD, THD, and RC1 are always administered in full.
Regardless of how they are obtained, MMPI-2-RF scores can be used for diagnosis and treatment planning. Of note, the authors are not suggesting that this information be relied upon exclusively to deny individuals surgery. Rather, MMPI-2-RF findings can point to areas of vulnerability that could affect outcomes and be addressed pre- and postoperatively with evidence-based interventions.

The major limitation of this study is the results reflect the performance of an FCA in a real-data simulation with existing MMPI-2-RF scores. Future research using actual administration conditions is needed as a next step toward possible implementation. It is possible that bariatric surgery patients have longer administration times than the 25 to 35 minutes projected by the MMPI-2-RF manual for the typical test-taker, which would increase administration time estimates for the FCA approach (but increase the absolute time savings relative to a standard administration). Future research is also needed to determine if a shorter administration method, such as an FCA, improves the likelihood that test-takers of lower literacy levels provide valid information on the test. It is also important to explore extra test indicators of psychopathology among individuals whose elevations would not have been caught by the FCA approach. Finally, future research could compare the FCA method to other abbreviated administration techniques such as item response theory (IRT). IRT can facilitate adaptive testing that provides an estimate of a latent trait measured by a scale. However, there are practical limitations of an IRT-based adaptive administration approach (e.g., practitioners' willingness to rely on latent trait estimates to make clinical decisions), and efforts to apply this technique to broadband personality inventories such as the MMPI have yet to produce viable measures.

\section{Conclusions}

Notwithstanding these limitations, this study is the first evaluation of an FCA of MMPI-2-RF in sample bariatric surgery candidates. The findings indicate that time savings of $44 \%$ to $88 \%$ are possible with minimal loss of information when using an FCA strategy. These time savings may increase the applicability of the MMPI-2-RF to presurgical evaluations of bariatric surgery patients, where research literature indicates the test can help identify psychological impediments to successful surgical outcomes.

\section{Disclosures}

Yossef S. Ben-Porath is a paid consultant to the MMPI2-RF publisher, the University of Minnesota Press, and distributor, Pearson Assessments. He receives royalties on sales of MMPI-2-RF materials and research grants from the MMPI-2-RF publisher. Anthony M. Tarescavage and Ryan J. Marek also receive research funding from the MMPI-2-RF publisher not related to this study. 
[1] Ben-Porath YS, Tellegen A. MMPI-2-RF: Manual for administration, scoring and interpretation, Minneapolis: University of Minnesota Press; 2008. 2011.

[2] Marek R, Heinberg L, Lavery M, Merrell JR, Ashton K. A review of psychological assessment instruments for use in bariatric surgery evaluations. Psychol Assess 2016;28:1142-57.

[3] Marek RJ, Ben-Porath YS, Sellbom M, McNulty JL, Heinberg LJ. Validity of Minnesota Multiphasic Personality Inventory - 2 - Restructured Form (MMPI-2-RF) scores as a function of gender, ethnicity, and age of bariatric surgery candidate. Surg Obes Rel Dis 2015;11:627-34

[4] Marek RJ, Ben-Porath YS, Windover A, et al. Assessing psychosocial functioning of bariatric surgery candidates with the Minnesota multiphasic personality inventory-2 restructured form (MMPI-2-RF). Obes Surg 2013;23:1864-73.

[5] Marek RJ, Tarescavage AM, Ben-Porath YS, Ashton K, Rish JM, Heinberg LJ. Using presurgical psychological testing to predict 1-year appointment adherence and weight loss in bariatric surgery patients: predictive validity and methodological considerations. Surg Obes Rel Dis 2015;11:1171-81.

[6] Tarescavage A, Windover A, Ben-Porath Y, et al. Use of the MMPI-2-RF suicidal/death ideation and substance abuse scales in screening bariatric surgery candidates. Psychol Assess 2013;25:1384-9.
[7] Fabricatore AN, Crerand CE, Wadden TA, Sarwer DB, Krasucki JL. How do mental health professionals evaluate candidates for bariatric surgery? Survey results. Obes Surg 2006;16:567-73.

[8] Jensen-Doss A, Hawley KM. Understanding barriers to evidence-based assessment: clinician attitudes toward standardized assessment tools. J Clin Child Adolesc Psychol 2010;39:885-96.

[9] Tarescavage A, Ben-Porath Y. Examination of the feasibility and utility of flexible and conditional administration of the Minnesota Multiphasic Personality Inventory-2-Restructured Form. Psychol Assess 2017;29:1337-48.

[10] Heinberg LJ, Ashton K, Windover A. Moving beyond dichotomous psychological evaluation: the Cleveland Clinic Behavioral Rating System for weight loss surgery. Sur Obes Rel Dis 2010;6:185-90.

[11] Tellegen A, Ben-Porath YS. Minnesota Mutliphasic Personality Inventory-2-Restructured Form: Technical manual, Minneapolis: University of Minnesota Press; 2008. 2011.

[12] Ben-Porath YS. Interpreting the MMPI-2-RF. Minneapolis: University of Minnesota Press; 2012.

[13] Rosenberger PH, Henderson KE, Grilo CM. Psychiatric disorder comorbidity and association with eating disorders in bariatric surgery patients: a cross-sectional study using structured interview-based diagnosis. J Clin Psychiatry 2006;67:1080-5.

[14] Melissa A, Kalarchian PD, Marsha D, et al. Psychiatric disorders among bariatric surgery candidates: relationship to obesity and functional health status. Am JPsychiatry 2007;164:328-34.

[15] Mitchell JE, Selzer F, Kalarchian MA, et al. Psychopathology before surgery in the longitudinal assessment of bariatric surgery-3 (LABS-3) psychosocial study. Surg Obes Relat Dis 2012;8:533-41.

[16] Walfish S. Reducing Minnesota Multiphasic Personality Inventory defensiveness: effect of specialized instructions on retest validity in a sample of preoperative bariatric patients. Surg Obes Rel Dis 2007;3:184-8. 